\title{
Publisher Correction: ERK1/2 phosphorylation predicts survival following anti-PD-1 immunotherapy in recurrent glioblastoma
}

Víctor A. Arrieta, Andrew X. Chen, J. Robert Kane, Seong Jae Kang, Cynthia Kassab, Crismita Dmello, Junfei Zhao, Kirsten B. Burdett, Pavan S. Upadhyayula, Catalina Lee-Chang, Joseph Shilati, Dinesh Jaishankar, Li Chen, Andrew Gould, Daniel Zhang (D), Jinzhou Yuan, Wenting Zhao, Xiaoyang Ling, Jared K. Burks (D), Brice Laffleur (D), Christina Amidei, Jeffrey N. Bruce (D), Rimas V. Lukas, Jonathan T. Yamaguchi, David Cieremans, Gerson Rothschild, Uttiya Basu (D), Matthew McCord, Daniel J. Brat, Hui Zhang, Lee A. D. Cooper D, Bin Zhang, Peter Sims, Tim F. Cloughesy (D), Robert Prins (D), Peter Canoll, Roger Stupp, Amy B. Heimberger (iD, Craig Horbinski (D), Fabio M. Iwamoto (D), Raul Rabadan (D) and Adam M. Sonabend (iD

Correction to: Nature Cancer https://doi.org/10.1038/s43018-021-00260-2, published online 29 November 2021.

In the version of this article initially published, there was an error in the author affiliations for Gerson Rothschild and Uttiya Basu. Both authors are affiliated with the Department of Microbiology and Immunology, Columbia University, New York, NY, USA, and not, as originally published, University of Rennes 1 . The affiliations have been updated in the HTML and PDF versions of the article.

Published online: 11 February 2022

https://doi.org/10.1038/s43018-022-00343-8

(C) The Author(s), under exclusive licence to Springer Nature America, Inc. 2022 